\title{
La vie et la pratique d'un notaire rural sous le régime français : le cas de Guillaume Barette, notaire à La Prairie entre 1709-1744
}

\section{Louis Lavallée}

Volume 47, numéro 4, printemps 1994

URI : https://id.erudit.org/iderudit/305277ar

DOI : https://doi.org/10.7202/305277ar

Aller au sommaire du numéro

Éditeur(s)

Institut d'histoire de l'Amérique française

ISSN

0035-2357 (imprimé)

1492-1383 (numérique)

Découvrir la revue

Citer cet article

Lavallée, L. (1994). La vie et la pratique d’un notaire rural sous le régime français : le cas de Guillaume Barette, notaire à La Prairie entre 1709-1744. Revue d'histoire de l'Amérique française, 47(4), 499-519.

https://doi.org/10.7202/305277ar

\section{Résumé de l'article}

Si nous utilisons fréquemment les archives qu'ils nous ont laissées, nous connaissons mal cependant les notaires d'Ancien Régime. Cet essai vise à étudier l'un d'eux, Guillaume Barette, qui a pratiqué pendant trente-cinq ans dans la seigneurie de La Prairie, l'une des plus peuplées de la région montréalaise au XVIII ${ }^{\mathrm{e}}$ siècle. Après l'avoir replacé dans son cadre spatial, nous avons reconstitué sa vie familiale et professionnelle et analysé sa fortune et son patrimoine. Nous avons finalement étudié le contenu de son greffe qui éclaire la pratique d'un notaire rural sous le Régime français. 


\title{
LA VIE ET LA PRATIQUE D'UN NOTAIRE RURAL SOUS LE RÉGIME FRANÇAIS: LE CAS DE GUILLAUME BARETTE, NOTAIRE À LA PRAIRIE ENTRE 1709-1744
}

\author{
LOUIS LAVALLÉE \\ Département d'histoire \\ Université de Montréal
}

\section{RÉSUMÉ}

Si nous utilisons fréquemment les archives qu'ils nous ont laissées, nous connaissons mal cependant les notaires d'Ancien Régime. Cet essai vise à étudier l'un d'eux, Guillaume Barette, qui a pratiqué pendant trente-cinq ans dans la seigneurie de La Prairie, l'une des plus peuplées de la région montréalaise au XVIII ${ }^{e}$ siècle. Après l'avoir replacé dans son cadre spatial, nous avons reconstitué sa vie familiale et professionnelle et analysé sa fortune et son patrimoine. Nous avons finalement étudié le contenu de son greffe qui éclaire la pratique d'un notaire rural sous le Régime français.

\section{ABSTRACT}

Although historians often use notarized contracts of the "Ancien-Régime», the notaries who drew them up are not well known. This essay is devoted to the study of one such notary, Guillaume Barette, who for thirty-five years practiced in the populous Montréal-area seigneury of La Prairie. After placing him in his spatial context, I have reconstituted his family and professional life and analyzed his fortune and inheritance. Finally, I have studied the acts that he produced, in order to shed light on the practices of a rural notary of the French regime.

Depuis une trentaine d'années, l'histoire sociale s'est principalement développée grâce à l'apport fécond des minutes notariales dont les vertus ont été sans cesse rappelées par les historiens soucieux de reconstituer le tissu social des populations d'Ancien Régime. L'intérêt porté par les historiens aux structures sociales, aux groupes sociaux, aux problèmes de classification et de stratification, à l'histoire sociale en général, fréquemment identifiée à une forme d'histoire totale, les a conduits à s'appuyer sur cette documentation qui se prêtait à l'histoire sérielle chère à Pierre Chaunu et pouvait leur fournir les matériaux nécessaires pour l'étude du social inscrit dans un cadre temporel de 
longue durée. À cet égard, la communication présentée par Ernest Labrousse, au Congrès international des Sciences historiques à Rome en $1955^{1}$, a marqué une date importante et fut l'acte fondateur des nombreux travaux qui allaient par la suite utiliser les minutes notariales jusque-là négligées ou ignorées par les historiens. Un fait demeure: depuis cette date, l'histoire sociale est devenue principalement tributaire de cette documentation, et l'expérience acquise par les historiens a donné lieu à un raffinement des méthodes d'utilisation et d'exploitation du corpus ${ }^{2}$. Que l'on songe seulement aux très nombreux textes méthodologiques proposés par les utilisateurs du contrat de mariage et de l'inventaire après-décès. Il ne paraît donc pas nécessaire d'évoquer de nouveau la richesse de la documentation notariée ni d'en rappeler les qualités qui sont depuis longtemps admises.

En revanche, nous sommes beaucoup moins renseignés sur les auteurs de ce corpus, les notaires eux-mêmes, qui n'ont fait l'objet que de très peu d'études. En effet, «un dénominateur commun transcende l'extraordinaire abondance des travaux fondés sur l'exploitation des archives notariales: l'absence ou plutôt l'occultation systématique du scripteur des documents sur lesquels s'appuient ces études: le notaire [...] force est de constater que nous ne savons pour ainsi dire rien de ce dernier, et à peine davantage de sa profession ${ }^{3}$ ». Plutôt que de souligner la contribution des actes notariés à l'histoire sociale, celle du Québec en particulier, nous avons donc préféré centrer notre recherche sur le notariat lui-même analysé à travers l'exemple de Guillaume Barette, l'un des plus importants notaires ruraux ${ }^{4}$ ayant pratiqué dans

1. Ernest Labrousse, «Voies nouvelles vers une histoire de la bourgeoisie occidentale aux XVIII ${ }^{e}$ et $\mathrm{XIX}^{\mathrm{e}}$ siècles (1700-1850)», $\mathrm{X}^{\mathrm{e}}$ Congrès international des Sciences historiques (Rome, 1955), Relazioni, Storia Moderna, IV: 365-396.

2. Il suffit, pour s'en convaincre, d'ouvrir le très imposant recueil de Jean-Paul Poisson, Notaires et société. Travaux d'histoire et de sociologie notariales (Paris, Économica, tomes III, 1985-1990), $736 \mathrm{p}$. et $597 \mathrm{p}$.

3. Notaires, notariat et société sous l'Ancien Régime, Études réunies et présentées par Jean L. Laffont (Toulouse, Presses universitaires du Mirail, 1990), 13-14.

4. Aussi bien en France qu'au Canada, notre ignorance du notariat rural est à peu près totale. Signalons parmi les quelques titres recensés dont aucun ne concerne le Régime français: Marie Bardet, "Activité notariale en milieu rural à l'époque moderne: essai de réflexion méthodologique», Jean L. Laffont, dir., Problèmes et méthodes d'analyse historique de l'activité notariale (XVe-XIX siècles) (Toulouse, Presses universitaires du Mirail, 1991), 29-41; id., «Notaires et société rurale en Haute-Auvergne (XVI ${ }^{e}-\mathrm{XVIII}^{\mathrm{e}}$ siècles)», Notaires, notariat..., 127138; Anne Fillon, «Notaires villageois et idées nouvelles. Le rôle du notaire rural dans l'évolution des mentalités», ibid., 119-125; Line Shorka, «Étude de l'activité notariale d'un notaire seigneurial tourangeau (1787-An VIII», Problèmes et méthodes..., 141-153; $M$. Toublanc, «Le notaire rural, intermédiaire entre les paysans et les propriétaires seigneuriaux et urbains: un type social des campagnes lyonnaises?», Les actes notariés. Source de l'histoire sociale (XVI ${ }^{e}$-XIXe siècles), Actes du colloque de Strasbourg (1978) réunis par Bernard Vogler (Strasbourg, Librairie Istra, 1979), 85-103; Michel Guénette, Les notaires de La Prairie 1760- 
la plaine de Montréal au XVIII ${ }^{\mathrm{e}}$ siècle. Le notaire Barette, dont nous allons suivre l'itinéraire et analyser le contenu du greffe, va nous permettre de saisir la teneur et l'importance de la pratique d'un notaire qui a exercé dans l'une des seigneuries les plus peuplées de la région montréalaise au XVIII ${ }^{e}$ siècle. En balisant les principales étapes de sa vie professionnelle et économique, nous serons également mieux renseignés sur la profession de notaire en Nouvelle-France et sur la place que ces officiers publics occupaient dans la vie sociale et seigneuriale au Canada. Cette étude s'appuie sur une abondante documentation de première main, registres paroissiaux, actes notariés, documents judiciaires, qui nous a permis de reconstituer la vie familiale et professionnelle de Barette, de mesurer son statut économique, de découvrir enfin le poids et la spécificité de sa pratique.

\section{1 - UN HABITANT DE LA PRAIRIE}

Situons d'abord le personnage dans le lieu où, pendant trente-cinq ans, il a exercé sa profession. Guillaume Barette habite le village de La Prairie sur la rive sud de Montréal. Ce bourg est au cœur de la seigneurie du même nom concédée aux jésuites en 1647. Cet immense territoire seigneurial qui couvre $120 \mathrm{~km}^{2}$ n'est toutefois occupé qu'à partir de 1667. En effet, il faut attendre l'expédition punitive de Tracy et la paix qui en résulte pour que la côte sud du gouvernement de Montréal s'ouvre à la colonisation. Ainsi, en 1667, La Prairie devient le premier espace colonisé par des Blancs sur la rive méridionale du fleuve, à la hauteur de Montréal. Jusqu'en 1701, la sécurité des lieux est cependant mal assurée malgré l'érection d'un fort par Gédéon de Catalogne en 1689. La population prend tout de même son envol et, à l'aube du XVIII e siècle, elle devait atteindre près de 350 habitants ${ }^{5}$. Libérée de la menace iroquoise depuis 1701, soutenue par l'immigration et la fécondité de sa population, La Prairie se développe ensuite à un rythme constant. La seigneurie des jésuites tire alors profit de sa position géographique très avantageuse qui lui permet de devenir le relais nécessaire, le lieu de passage obligé entre Montréal et la vallée du Richelieu ouverte à la colonisation depuis que sa sécurité est garantie par une ligne de forts dont La Prairie constitue la tête de pont. À la Conquête, sa population tourne autour de 2000 habitants et son

1850. Étude socio-économique, mémoire de maîtrise (histoire), Université de Montréal, 1992, 172 p.; Hélène Lafortune, La situation de la profession notariale à l'Assomption entre 1800 et 1850, mémoire de maîtrise (histoire), Université de Montréal, 1981, 190 p.

5. Pour tous les détails qui concernent l'histoire de La Prairie, nous renvoyons à notre livre: Louis Lavallée, La Prairie en Nouvelle-France, 1647-1760: étude d'histoire sociale (Montréal et Kingston, McGill-Queen's University Press, 1992), 304 p. 
village est l'un des plus importants du gouvernement de Montréal. Plusieurs éléments reflètent l'accroissement du nombre des hommes au XVIII ${ }^{e}$ siècle. La première église de pierre est construite en 1705 , le village est agrandi en 1724 et la carte paroissiale redessinée en 1722 et 1744. Autre témoignage enfin de cette augmentation de population, signe de temps économiques et démographiques nouveaux, les notaires qui avaient fui La Prairie à la fin du XVII ${ }^{e}$ siècle $^{6}$ y reviennent au XVIII ${ }^{\mathrm{e}}$ siècle quand Guillaume Barette ouvre son étude en 1709 et se trouve relayé en 1745 et 1753 par André Souste et Joseph Lalanne. En trente-cinq ans d'exercice, soit de 1709 à 1744, Barette est donc le seul notaire de la seigneurie, et il dessert une population qui passe en gros de 450 à 1500 personnes établies à l'intérieur d'un espace couvrant à peu près $120 \mathrm{~km}^{2}$.

Avant d'ouvrir son greffe, dressons une biographie sommaire de Barette (elle n'a jamais été faite) en isolant les principaux événements qui ont marqué sa vie familiale et professionnelle et en reconstituant l'histoire de sa modeste fortune entre le moment du mariage et celui où elle a été partagée.

Guillaume Barette est né en $1680^{7}$ à Cap-de-la-Madeleine, seigneurie appartenant aux jésuites. Il était le huitième enfant de Guillaume Barette et de Louise Charrier, tous deux originaires de France, de Normandie et du Poitou. De l'apprentissage et de la formation du futur notaire, nous ne savons presque rien. Un acte déposé aux Archives nationales du Québec à Trois-Rivières nous apprend que Guillaume Barette s'était engagé pour deux ans auprès du notaire montréalais Antoine Adhémar pour lui servir de clerc ${ }^{8}$. Le contrat est cependant annulé le 2 juillet 1699 sans qu'il soit possible de savoir si Barette a réellement travaillé chez Adhémar. Quoi qu'il en soit, Antoine et Jean-Baptiste Adhémar vont devenir les notaires de Guillaume Barette qui leur a demandé de rédiger tous ses actes personnels. Sans doute appelé par les jésuites, seigneurs de La Prairie de la Madeleine, il s'y marie le 20 juin 1706 dans la nouvelle église de pierre érigée un an plus tôt par le curé Vilermaula, sulpicien pugnace

6. Au XVII ${ }^{e}$ siècle, trois notaires ont exercé à La Prairie. Joseph Tissot, secrétaire des jésuites, est le plus important et il a rédigé quelque 180 contrats entre 1671 et 1681 . Pierre Colard et Laurent Hervieux ne signent que très peu d'actes en 1686 et 1690 . Au-delà de cette date, il n'y a aucun notaire jusqu'à l'arrivée de Barette, et les habitants du lieu doivent traverser le fleuve pour faire rédiger leur contrats.

7. L'âge qui apparaît au contrat de mariage et au registre de sépulture nous oblige à revoir la date de sa naissance (1678) indiquée par René Jetté dans son Dictionnaire généalogique des familles du Québec des origines à 1730 (Montréal, Les Presses de l'Université de Montréal, 1983), 48.

8. ANQTR, Jean Cusson, 12 février 1698, engagement de Guillaume Barette par Antoine Adhémar. 
à l'origine d'une querelle considérable qui a opposé jésuites et sulpiciens et bouleversé la vie des paroissiens au début du XVIII ${ }^{e}$ siècle. Obéissant à une coutume suivie par presque tous les habitants du lieu', Guillaume Barette avait demandé, trois jours plus tôt, au notaire Adhémar de Montréal de rédiger son contrat de mariage ${ }^{10}$. Le document n'est pas très bavard et va à l'essentiel. Le futur notaire, âgé de 26 ans, s'allie à Jeanne Gagné, de quatre ans sa cadette. Elle était la fille de Pierre Gagné, l'un des plus anciens habitants de la seigneurie et l'une des figures dominantes de sa jeune histoire puisqu'il avait occupé les fonctions de marguillier et de capitaine de milice. Fait singulier dans une société paysanne en majorité analphabète, les deux futurs époux savent signer de même que les parents de Jeanne Gagné. Une douzaine de témoins assistent à la lecture du contrat qui spécifie le régime de la communauté de biens, fixe le douaire à 600 livres, le préciput à 400 livres, mais reste muet (comme c'est trop souvent le cas dans ce type de document) sur le montant de la dot et sur l'apport du futur époux. Le destin familial du couple Barette-Gagné n'est pas différent de celui de beaucoup d'autres couples de la seigneurie. En treize ans d'union, soit de 1706 à 1719, ils ont eu neuf enfants dont sept ont survécu et se sont mariés. En 1719, le mariage est rompu par le décès de Jeanne Gagné qui est emportée, précise le registre mortuaire, par une «longue maladie» quatre mois après avoir donné naissance à son dernier enfant, Augustin. Devenu veuf à 39 ans, le notaire ne se remariera pas; il décède en 1745 à l'âge de 64 ans.

\section{2 - PATRIMOINE, FORTUNE ET STATUT ÉCONOMIQUE}

Les actes notariés que nous avons réunis, tous déposés chez les notaires Adhémar, père et fils, nous permettent de reconstituer l'histoire patrimoniale de Guillaume Barette et de mesurer le niveau de sa fortune. En 1709, les jésuites lui concèdent, au bourg de La Prairie, un emplacement, rue Saint-François-Xavier ${ }^{11}$, qu'il va occuper jusqu'à sa mort. L'inventaire de ses biens que l'on dresse en $1724^{12}$ nous apprend qu'il y habite une vieille maison bâtie de pièces sur

9. À La Prairie, le mariage est habituellement précédé de la visite chez le notaire. Entre 1670 et 1759, 558 mariages ont été bénis par les curés des trois paroisses de la seigneurie et 507 contrats de mariage ont été rédigés, soit une proportion de $90,9 \%$ entre l'acte civil et religieux.

10. ANQM, Antoine Adhémar, 17 juin 1706, contrat de mariage entre Guillaume Barette et Jeanne Gagné. Barette.

11. ANQM, Antoine Adhémar, 21 mars 1709, concession par les jésuites à Guillaume

12. ANQM, Jean-Baptiste Adhémar, 5 juillet 1724, inventaire des biens de Guillaume Barette. 
pièces, couverte de paille et dont la cheminée et la cave sont en maçonnerie. Cette maison, semblable à celles que l'on retrouve habituellement dans la seigneurie ${ }^{13}$, mesure $35 \times 20$ pieds et comprend, en plus de la cave, une chambre où le notaire travaille, une cuisine et un grenier. Dans cet espace, vivent le veuf et ses sept enfants, tous célibataires au moment où est établi l'inventaire après-décès. Le document précise en outre que la communauté Barette-Gagné, qui sera dissoute le lendemain lors du partage des biens ${ }^{14}$, comprend une vieille grange en poteaux, entourée de pieux, couverte de paille et mesurant $30 \times 22$ pieds, une étable de pièces sur pièces de 20 pieds $^{2}$, également couverte de paille et, finalement, un petit fournil de même facture couvert d'écorces, mesurant $8 \times 10$ pieds.

Le détail de l'habitation, la présence d'instruments aratoires (une charrue et une herse) et de cheptel ( 3 vaches, 2 veaux, 2 bœufs de «tir», 2 jeunes bœufs, 2 taureaux, 2 vieux chevaux, 3 cochons, 2 agneaux et 4 brebis) nous éclairent sur les activités agricoles de Barette qui s'ajoutent à celle de notaire. En effet, il est en même temps agriculteur et jouit du revenu de ses terres qu'il n'a sans doute jamais fait valoir lui-même mais qu'il a peut-être affermées au début et dont l'exploitation sera plus tard confiée à ses trois fils. Dans l'inventaire préparé par Adhémar en 1724, apparaissent trois terres dont deux sont en partie défrichées. La première, de 90 arpents, concédée le même jour ${ }^{15}$ que l'emplacement au village, est partagée entre les seigneuries jumelles de La Prairie et du Sault-Saint-Louis. Elle appartient à la côte Sainte-Catherine qui occupe, en bordure du fleuve, le site de l'ancien village iroquois récupéré par les jésuites après la migration des Indiens et loti en concessions distribuées à leurs amis et leurs plus fidèles censitaires. La seconde, située à la côte Saint-Joseph, dans la seigneurie de La Prairie, sur le territoire de la future paroisse Saint-Philippe, a une superficie de 80 arpents et sa concession date de $1714^{16}$. La dernière, qui couvre 90 arpents à la côte Saint-François-Xavier, près de la rivière de la Tortue, a été achetée par Barette trois ans plus tard ${ }^{17}$.

13. Kalm qui visite la seigneurie de La Prairie en 1749 écrit: «Les maisons de petite taille, sont construites en bois [...]. Le toit est très incliné et couvert de chaume.»Voyage de Pehr Kalm au Canada en 1749, traduction annotée du journal de route par Jacques Rousseau et Guy Béthune (Montréal, Pierre Tisseyre, 1977), 177. Barette.

14. ANQM, Jean-Baptiste Adhémar, 6 juillet 1724, partage des biens de Guillaume Barette.

15. ANQM, Antoine Adhémar, 21 mars 1709, concession par les jésuites à Guillaume Barette.

16. ANQM, Jean-Baptiste Adhémar, 9 juin 1714, concession par les Jésuites à Guillaume

17. ANQM, Jean-Baptiste Adhémar, 22 mars 1717, vente par Moïse Dupuis à Guillaume Barette. 
Ainsi, en treize ans de communauté, le notaire et sa femme ont donc acquis 260 arpents de terre. À une époque où le réservoir de terres à concéder est encore immense dans la seigneurie de La Prairie, ce total est conforme à celui que possèdent de nombreux ménages paysans. C'est celui que se partagent par part égale, en 1724, le veuf et ses sept enfants mineurs.

La coutume de Paris qui commande la division égale des biens entre les héritiers (une part va au conjoint survivant et l'autre aux enfants) conduit alors à un émiettement du patrimoine ${ }^{18}$ que tentent, tant bien que mal, de reconstituer, en partie ou dans son intégralité, les bénéficiaires du partage. Les manières adoptées par la famille Barette pour recréer le bien familial sont semblables à celles qu'ont retenues la plupart des familles paysannes de la seigneurie ${ }^{19}$. Elles apparaissent à travers les nombreux contrats de vente et d'échange dont la réunion permet d'identifier les agents du remembrement et de suivre les stratégies qu'ils ont déployées. L'un des gendres, Pierre Bourdeau, récupère la terre de Saint-Joseph et le cadet de la famille, Augustin, regroupe celle de Sainte-Catherine, la plus importante et la plus estimée ${ }^{20}$. Au moment où survient le décès de Guillaume Barette en 1745, le dernier-né, Augustin, est le mieux placé pour rassembler la concession de la côte Sainte-Catherine. Il est alors le seul célibataire parmi les enfants du notaire qui, à la date de sa mort, sont tous mariés et établis depuis plusieurs années. Il s'était par ailleurs engagé depuis quelques années dans la traite aux Pays d'en-haut, et cette activité saisonnière lui avait fourni les moyens de racheter les parcelles de terre vendues par les cohéritiers. À cet égard, la famille Barette ne faisait pas exception. Au même titre que la plupart des autres familles de la seigneurie des jésuites, elle participait, par la voie de l'engagement, à l'activité commerciale la plus importante dans l'économie de la Nouvelle-France ${ }^{21}$.

18. Émiettement particulièrement marqué dans le cas de la succession Barette qui a connu trois partages. ANQM, Jean-Baptiste Adhémar, 5 juillet 1724; ANQM, Jean-Baptiste Adhémar, 18 octobre 1743; ANQM, André Souste, 30 septembre 1745.

19. Louis Lavallée, La Prairie..., 182-214.

20. La terre de la rivière de la Tortue ne fait plus partie du patrimoine depuis 1734. Elle a été rétrocédée aux jésuites par le notaire Barette (ANQM, Jean-Baptiste Adhémar, 9 juillet 1734, rétrocession par Guillaume Barette) au moment où l'intendant Hocquart applique avec une vigueur nouvelle les Arrêts de Marly qui toucheront plusieurs habitants de La Prairie dont les terres seront réunies au domaine à partir de 1732. Quant à l'emplacement au village, les héritiers le vendent (ANQM, Jean-Baptiste Adhémar, 8 février 1745, vente par les héritiers Barette à Joseph Bruneau) un mois après le décès du notaire.

21. Louis Lavallée, La Prairie..., 215-230. Pierre, le fils aîné de Guillaume Barette, a aussi effectué comme engagé quelques voyages aux Pays d'en-haut. 
S'il est permis, grâce à l'abondante documentation laissée par les notaires, de reconstituer, parfois très loin dans le détail, l'histoire patrimoniale de la famille Barette, il est impossible, en revanche, de refaire l'historique de sa fortune et d'en suivre les étapes. À l'instar de la plupart des fortunes paysannes de La Prairie, elle offre l'image d'une banalité peu diserte et d'une honnête médiocrité dont les silences de la documentation rendent compte. L'inventaire des biens de la communauté Barette-Gagné dressé par le notaire Adhémar en 1724 , cinq ans après le décès de l'épouse de Guillaume Barette, demeure le seul document «quantitatif» qui nous permet de juger de son contenu et de mesurer son niveau. La compilation que nous avons effectuée de tous les effets prisés à l'inventaire se lit ainsi:

\section{TABLEAU 1 \\ Inventaire des biens de la communauté Barette-Gagné}

\begin{tabular}{|c|c|}
\hline \multirow{2}{*}{$\begin{array}{l}\text { Biens meubles } \\
\text { Cheptel }\end{array}$} & $\begin{array}{l}414 \text { livres } \\
355 \text { livres }\end{array}$ \\
\hline & $\frac{355 \text { livres }}{769 \text { livres }}$ \\
\hline Dettes 1 & 283 livres \\
\hline Total & 486 livre \\
\hline
\end{tabular}

Ces renseignements laconiques sont cependant éloquents. Certes, le bilan de la fortune de Barette est positif mais son niveau situe le notaire très bas dans la hiérarchie économique que nous avons découverte à La Prairie ${ }^{22}$. Avec ses 486 livres d'actif, Guillaume Barette rejoint les paysans les plus médiocres de la seigneurie. La valeur de son mobilier et de son cheptel, son endettement auprès des marchands montréalais, son avoir total enfin le classent aux derniers échelons de cette société à majorité paysanne. Il n'a aucun argent liquide, aucun bijou, aucune vaisselle d'argent et pas un livre n'est recensé dans l'inventaire ${ }^{23}$. Il ne détient pas davantage ce pouvoir économique qui nous aurait été révélé par la présence dans le document des dettes actives. On pourrait objecter que le total de la fortune de Barette est très relatif puisque nos calculs ne reposent que sur la

22. Ibid., 247.

23. Cela ne doit pas nous étonner. Les notaires ruraux ne possédaient pas habituellement de bibliothèque. Les seuls livres dont ils avaient besoin étaient ces manuels leur servant de modèle pour la préparation des contrats et qui, à cause de leur faible valeur, n'étaient pas toujours prisés. 
seule valeur des avoirs mobiliers et ignorent complètement le patrimoine foncier. En toute justice et pour être plus exact, il faudrait donc tenir compte des biens immeubles dans la constitution du bilan. Or, comme c'est le cas habituellement dans les inventaires, les terres de Barette ne sont pas évaluées. Même si elles l'étaient, cet ajout serait illusoire car vendre ses terres pour convertir en argent quelques actifs, c'était se défaire d'une partie de son gagne-pain, de ce qui assurait à la famille une existence relativement indépendante ${ }^{24}$.

Dix-huit ans après son mariage, Barette ne s'était guère enrichi. La faiblesse de ses honoraires et le poids de sa nombreuse famille le condamnaient sans doute à occuper la base de la pyramide économique de La Prairie. Son statut était celui de bien d'autres notaires en Nouvelle-France. André Vachon a écrit qu'en général l'exercice de leur profession ne leur permettait pas de vivre de façon convenable ${ }^{25}$. Il ajoutait que le prestige qu'ils auraient pu tirer de leur fonction était «nettement contrebalancé par un train de vie généralement misérable ${ }^{26}$ » qui les classait souvent en deça de l'habitant ou du petit marchand. Il faudrait cependant nuancer cette affirmation et distinguer un notaire rural comme Barette d'un notaire urbain comme JeanBaptiste Adhémar qui a exercé à Montréal pendant près de quarante ans. Compte tenu des milieux dans lesquels ils pratiquaient, des activités si différentes générées par la ville et le plat pays, de la composition distincte de leur clientèle, il paraît difficile de confondre dans un même groupe deux officiers publics très éloignés l'un de l'autre. Le premier, agriculteur et notaire, desservait une population rurale très homogène et rédigeait à peu près toujours le même type d'actes qui ne concernaient que l'histoire interne de la seigneurie. Le second, montréalais d'appartenance, rejoignait une clientèle très diversifiée et était appelé à rédiger des contrats qu'ignoraient les notaires ruraux. La majorité des opérations de crédit (obligations, rentes, etc.) et commerciales (sociétés, accords divers, congés et engagements pour les Pays d'en-haut, etc.), en particulier, lui revenaient habituellement. Quoi qu'il en soit de ces différences de statut que l'on ne saurait négliger, il semble cependant, si l'on suit André Vachon, que la profession était mal rémunérée ${ }^{27}$ et que la situation économique du notaire en Nouvelle-France n'était pas des plus heureuses. En témoignent la cor-

24. Louis Michel, «Aperçus sur l'endettement en milieu rural au XVIII' siècle: l'exemple de Varennes et Verchères». Communication présentée au congrès de l'Institut d'histoire de l'Amérique française, Ottawa, 1979. (Inédit)

25. André Vachon, Histoire du notariat canadien 1621-1960 (Québec, Les Presses de l'Université Laval, 1962), 45.

26. Ibid., 46.

27. Ibid., 47. 
respondance officielle qui, fréquemment, déplore cet état de fait, les suppliques des notaires eux-mêmes adressées à l'intendant à qui on réclame de l'aide ${ }^{28}$, le cumul des charges (greffier, arpenteur, huissier, médecin, commerçant, procureur fiscal ou juge d'une seigneurie, etc. ${ }^{29}$ ) souvent rendu nécessaire par la faiblesse des honoraires, le recours à des expédients, enfin, auxquels on peut sans doute rattacher l'épisode suivant, très singulier dans la vie du notaire Barette. Connaissant la situation matérielle de Guillaume Barette, il revêt une signification particulière à la lumière de ce que nous savons déjà sur l'état médiocre de sa fortune.

\section{3 - UN NOTAIRE CONTREBANDIER?}

En 1718, une dizaine d'habitants de La Prairie, dont Guillaume Barette, sont soupçonnés par l'intendant Begon ${ }^{30} \mathrm{~d}$ 'avoir été à Albany, quelquefois appelé Orange dans les textes en souvenir de la domination hollandaise. Accusés d'avoir pratiqué la traite illégale des fourrures, les prévenus sont soumis à l'interrogatoire de Pierre Raimbault, procureur du roi à Montréal et subdélégué de l'intendant, qui a reçu de Bégon le mandat de faire enquête et de mener l'instruction.

Depuis longtemps, La Prairie était un haut lieu de la traite légale et illicite ${ }^{31}$. Sa géographie, d'abord, l'invitait naturellement à participer à cette activité économique à laquelle elle restera longtemps attachée, même au-delà du Régime français. La seigneurie des jésuites est au carrefour d'un réseau hydrographique, à la croisée des voies d'eau qui, en direction ouest (par le fleuve) et sud (par le Richelieu et l'Hudson), conduisent aux Pays d'en-haut et à Albany, points d'arrivée du commerce licite et illégal. Cet impératif géographique explique à lui seul la participation prononcée de La Prairie au commerce des fourrures qui devait être, à la veille de la Conquête, l'une des plus importantes au sein du monde rural de la région montréalaise. Ce motif aurait pu à lui seul éclairer l'étendue de la contrebande dans cette région et faire comprendre l'audace des habitants de La Prairie et la tentation à laquelle plusieurs n'ont pas su résister. Mais il se trouve conforté par un second facteur qui a également encouragé le commerce interdit. Il faut rappeler ici la présence à La Prairie d'une mission iroquoise qui, progressivement, va gagner le fief voisin du Sault-Saint-Louis et dont on ne peut nier l'implication, la complicité

28. Ibid., 45 .

29. Ibid., 42-44.

30. ANQQ, Ordonnance de l'intendant Bégon du 8 juillet 1718.

31. Louis Lavallée, La Prairie..., 215-238. 
avec les Français quelquefois, dans la contrebande. Le voisinage des deux communautés et la tolérance manifestée par les autorités coloniales à l'égard des Iroquois a pendant longtemps nourri la traite illégale à laquelle, liés à l'occasion par des intérêts communs, Blancs et Indiens ont participé conjointement.

C'est dans ce contexte que s'est développée l'affaire à laquelle a été mêlé le notaire Barette. En septembre 1718, Pierre Raimbault l'interroge ${ }^{32}$ pour tenter de préciser son rôle dans cet acte de contrebande puni d'emprisonnement et d'une forte amende. Le procureur du roi lui demande avec qui il a voyagé au printemps de 1718 , où il est allé, combien de temps il s'est absenté et qui était le maître du canot? Le notaire réfute bien sûr l'accusation, nie avoir été à Orange et justifie son absence de La Prairie de la façon suivante. Il déclare à l'enquêteur avoir quitté son domicile un peu plus de trois semaines et s'être rendu à Cap-de-la-Madeleine (son lieu de naissance), en canot, avec Claude Bisaillon de La Prairie, pour y régler avec son frère des affaires concernant une terre dont il était propriétaire. Selon sa déclaration, il y aurait séjourné deux semaines, mais il ne peut fournir la preuve ni de son déplacement, ni de son séjour à l'extérieur de la seigneurie. En effet, il aurait couché dehors, sur la grève, à l'aller et au retour, et ne serait entré dans aucune maison. Immobilisé à cause d'une foulure au pied, il serait resté chez son frère, sans bouger, pendant deux semaines et, de ce fait, aucun témoin ne l'aurait aperçu. Finalement, le notaire exprime son indignation au procureur en lui faisant savoir «que dès le moment qu'un habitant est deux jours absent on l'accuse d'y avoir été [à Orange] ${ }^{33}$ ». Raimbault décide alors «d'informer plus amplement sur Barette ${ }^{34} \gg$ mais, faute de preuves, il doit libérer le notaire.

Nous ne saurons sans doute jamais si Guillaume Barette s'est rendu à Albany. Une chose est sûre cependant. Il s'est vraiment absenté de La Prairie au mois de mai 1718 puisqu'il n'a signé aucune minute pendant ce temps. En effet, il y a un trou de trois semaines dans son greffe à ce moment-là qui n'est pas habituel dans sa pratique à cette période de l'année. Où était-il? À Cap-de-la-Madeleine (son témoignage paraît douteux) ou chez les Anglais pour se refaire une fortune en participant à un commerce lucratif. Quoi qu'il en soit, s'il est vraiment allé à Albany, il n'a pas eu cette audace une seconde fois et jamais plus, après 1718 , le notaire Barette ne sera inquiété par la justice royale.

32. ANQM, Pièces détachées à caractère judiciaire, 13 août 1718.

33. Ibid.

34. Ibid. 


\section{4 - UN NOTABLE DE LA SEIGNEURIE}

Malgré sa piètre fortune, Guillaume Barette était l'un des notables de la seigneurie. De par sa fonction, il détenait:

un pouvoir certain, un pouvoir culturel lié au savoir, un pouvoir au service de ce qui [...] [était] sa raison d'être: organiser la circulation des biens et de l'argent et la redistribution du patrimoine. Comment cet homme aurait-il pu exercer une telle responsabilité sans avoir une influence, si mince soit-elle, sur la façon de régler successions, mariages, ventes, prêts, etc...? Sans devenir aux yeux de ses contemporains un homme de consei ${ }^{35}$ ?

Il aimait se faire appeler «Sieur Courville» et possédait son banc à l'église ${ }^{36}$. Quand le procureur Collet se présente à La Prairie, le 23 février 1721, pour enquêter sur le district des paroisses, il est cité dans le procès-verbal de l'assemblée des habitants immédiatement après Armand Ulric, curé du lieu, Clément Lériger, écuyer, seul noble qui habite la seigneurie, François Leber et Antoine Boyer, officiers de milice $^{37}$. L'une des seules personnes réellement alphabétisées à La Prairie, le notaire Barette était de ce fait souvent appelé à servir d'intermédiaire entre les habitants, les seigneurs et les autorités coloniales. Les jésuites faisaient souvent appel à ses services pour rédiger les contrats qui concernaient l'administration seigneuriale. En 1721, par exemple, il dresse le recensement que lui présentent les seigneurs de La Prairie ${ }^{38}$. À maintes reprises, il convoque, à leur demande, à l'issue de la messe paroissiale, l'assemblée des habitants qui à l'occasion se réunit dans sa maison pour discuter des affaires internes de la seigneurie ${ }^{39}$. En d'autres circonstances, il sert de porteparole à l'administration coloniale qui passe par lui pour faire connaître aux habitants ses dernières directives. Ainsi, en 1724, Barette est invité à lire à la porte de l'église de La Prairie l'ordonnance de Bouat, lieutenant général au tribunal de la juridiction royale de Montréal, qui oblige les censitaires à se réunir tous les deux ans pour élire les syndics de la commune ${ }^{40}$.

35. Marie Bardet, «Activité notariale...», 30.

36. ANQM, Guillaume Barette, 20 décembre 1726, contrat d'un banc par le curé à Guillaume Barette.

37 Mathieu-Benoît Collet, «Procès-verbaux sur la commodité et incommodité dressés dans chacune des paroisses de la Nouvelle-France [...]», Rapport de l'archiviste de la province de Québec (1921-1922): 304.

38. ANQM, Guillaume Barette, 29 septembre 1721, recensement de la seigneurie de La Prairie.

39. Voir pour exemple: ANQM, Guillaume Barette, 25 janvier 1722, assemblée des habitants de La Prairie.

40. ANQM, Guillaume Barette, 12 juin 1724, lecture d'une ordonnance de Bouat. 
Cette fonction de mandataire qu'on lui demandait de remplir à l'occasion n'était pas toujours sans danger, et Guillaume Barette n'a sans doute jamais oublié les événements douloureux qu'il a vécus en 1728. Cette année-là, le premier dimanche de mars, le notaire se présente à la sortie de la messe, devant l'église de La Prairie, pour lire et publier un ordre du roi défendant «de faire usage d'étoffe des Indes en cette colonie ${ }^{41}{ }^{1}$. Au même moment, il est assailli par plusieurs femmes dans la quarantaine assemblées à la porte de l'église pour protester contre la publication d'un édit qui risquait, selon elles, de leur enlever les indiennes qu'elles portaient. Elles le saisirent, lui bandèrent les yeux sans lui faire aucune violence cependant, lui arrachèrent le texte des mains et tour à tour le déchirèrent en plusieurs morceaux. Averties du geste que devait poser Barette, les femmes s'étaient réunies avant d'entrer dans l'église pour décider de la forme que devait prendre leur action qui n'avait rien d'improvisée. Elles nièrent malgré tout que leur agression fût préméditée, ne reconnurent pas l'assemblée et déclarèrent finalement ignorer qu'il s'agissait d'un ordre royal. À part cet épisode particulier, la vie professionnelle du notaire Barette semble avoir été paisible et, en trente-cinq ans, elle n'a été perturbée par aucun autre événement. Le moment est donc venu d'ouvrir son greffe et d'en étudier la composition.

\section{5 - LE CONTENU DE SON GREFFE}

Guillaume Barette appartient à cette minorité de notaires qui sous le Régime français sont Canadiens d'origine ${ }^{42}$. Il reçoit en 1708 de l'intendant Raudot les provisions de notaire et d'huissier pour exercer dans la seigneurie de La Prairie. Un an plus tard, la commission est élargie à toutes les seigneuries de la côte sud de Montréal où il n'y a ni notaire ni huissier d'établi43 ${ }^{43}$. En 1711, il renonce à ses fonctions d'huissier qu'il ne peut plus occuper «à cause de ses affaires domestiques ${ }^{44} \gg$. Il a pratiqué comme notaire à La Prairie pendant trente-cinq ans, n'a à peu près jamais débordé ce territoire et il a signé, entre le 23 décembre 1709 et le 24 décembre 1744 , un total de $1593^{45}$ actes qui, selon les années, se répartissent comme suit:

41. ANQM, Pièces détachées à caractère judiciaire, 15 mars 1728.

42. Selon André Vachon (Histoire du notariat..., 38), seulement $28,5 \%$ des notaires du Régime français sont nés au Canada.

43. ANQM, Antoine Adhémar, 2 novembre 1711, déclaration de Guillaume Barette. Selon ce document, la première commission date du 27 octobre 1708 et la seconde du 31 octobre 1709.

44. Ibid.

45. Ce chiffre est le résultat de la compilation que nous avons effectuée à la suite du dépouillement sys.tématique du greffe de Barette. 


\section{TABLEAU 2 \\ Répartition annuelle des actes de Barette}

$\begin{array}{rrrr}1709 & 1 & 1728 & 40 \\ 1710 & 17 & 1729 & 39 \\ 1711 & 21 & 1730 & 74 \\ 1712 & 20 & 1731 & 56 \\ 1713 & 18 & 1732 & 30 \\ 1714 & 30 & 1733 & 37 \\ 1715 & 22 & 1734 & 42 \\ 1716 & 31 & 1735 & 47 \\ 1717 & 53 & 1736 & 49 \\ 1718 & 44 & 1737 & 44 \\ 1719 & 31 & 1738 & 56 \\ 1720 & 31 & 1739 & 50 \\ 1721 & 44 & 1740 & 63 \\ 1722 & 45 & 1741 & 53 \\ 1723 & 78 & 1742 & 54 \\ 1724 & 57 & 1743 & 73 \\ 1725 & 85 & 1744 & 51 \\ 1726 & 53 & \text { Total } & \mathbf{1 5 9 3} \\ 1727 & 54 & & \end{array}$

Il a rédigé en moyenne 45,5 minutes par année en desservant une population qui, entre 1709 et 1744, est passée en gros de 450 à 1500 habitants. Les premières années, au cours desquelles il organise son étude, l'occupent moins. Entre 1709 et 1716, il ne signe en moyenne qu'une vingtaine d'actes par an. Son greffe se gonfle ensuite (1717$1744)$ et présente dans l'ensemble une remarquable stabilité $(51,1$ actes par an en moyenne) liée, on le verra, à un type de pratique dont le contenu n'a pas tellement varié. Seulement quelques années fastes $\left(1723,1725,1730,1743^{46}\right)$ ou creuses $\left(1719,1720,1732,1733^{47}\right)$ apparaissent et brisent un rythme qui demeure à peu près constant. Exprimée en mois, l'activité de Barette est cependant plus variable et certaines périodes de l'année l'accaparent davantage que d'autres. Les 1589 minutes dont nous connaissons le mois de rédaction s'ordonnent de la façon suivante:

46 À ces dates, le mouvement de la terre (ventes et concessions surtout) est particulièrement agité.

47. En 1732 et 1733 , en particulier, l'application plus sévère par Hocquart des Arrêts de Marly a contraint les jésuites à se montrer davantage économes dans la distribution de nouvelles terres. Le notaire Barette rédige alors moins d'actes de concession. 


\section{TABLEAU 3}

Répartition mensuelle des actes de Barette

$\begin{array}{lr}\text { Janvier } & 123 \\ \text { Février } & 114 \\ \text { Mars } & 139 \\ \text { Avril } & 127 \\ \text { Mai } & 165 \\ \text { Juin } & 177 \\ \text { Juillet } & 167 \\ \text { Août } & 85 \\ \text { Septembre } & 111 \\ \text { Octobre } & 122 \\ \text { Novembre } & 165 \\ \text { Décembre } & 94 \\ \text { Total } & 1589\end{array}$

Transcrite sur un graphique, la courbe du mouvement saisonnier présente des creux en août et en décembre et des sommets en mai, juin, juillet et novembre.

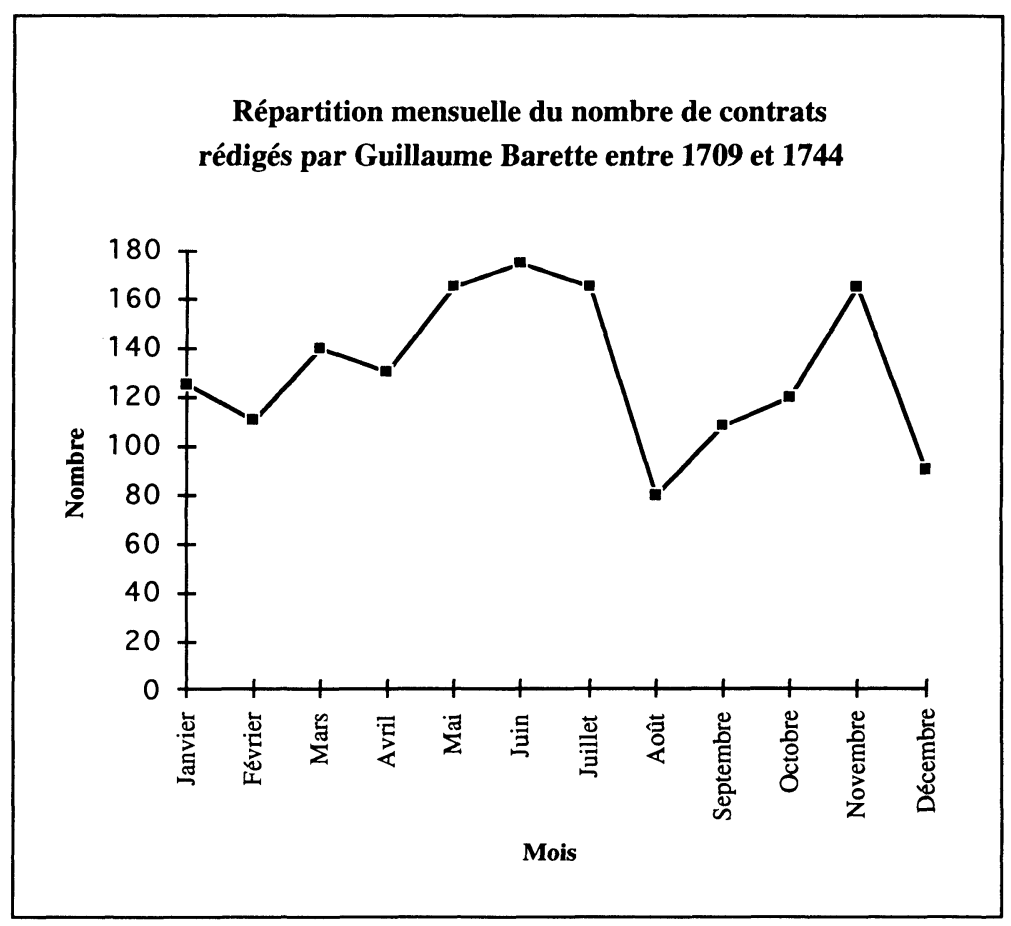


Pour interpréter les oscillations de la courbe, comprendre les pointes et les dépressions, il faut se rappeler que la société à laquelle appartenait Barette était essentiellement religieuse et rurale, paysanne et seigneuriale, que la religion et l'agriculture conditionnaient son mode de vie et déterminaient le calendrier de ses activités. Ainsi, la pratique du notaire Barette, alimentée en presque totalité par une population paysanne et catholique, se conformait à des impératifs agraires et religieux qui ont influencé le rythme mensuel de son activité. Ainsi, sa pratique était plus forte le printemps et l'été (de mai à juillet) alors que la société agricole et seigneuriale était en pleine effervescence et que les jésuites devenaient plus actifs, en particulier dans la concession des terres. Cependant elle était considérablement ralentie en août, au moment de la fenaison, lorsque les paysans, occupés aux travaux des champs, y concentraient le plus clair de leur temps. Par contre, Guillaume Barette rédigeait moins d'actes l'automne et l'hiver, surtout en décembre, période creuse de la nuptialité soumise à l'interdit de l'Avent que respectaient en général les habitants. Seule exception pendant ces mois, celui de novembre, mois fort de la nuptialité dans cette société d'Ancien Régime, où le notaire Barette était appelé à rédiger beaucoup de contrats de mariage.

L'analyse détaillée du contenu de son greffe éclaire le type de pratique d'un notaire rural en Nouvelle-France dont le champ d'action était passablement différent de celui des notaires urbains les plus importants. Les 1593 actes que Barette a signés entre 1709 et 1744 se répartissent selon l'ordre décroissant suivant:

\section{TABLEAU 4 \\ Composition du greffe de Barrette}

Concessions de terres

402

Ventes de terres et de droits successifs immobiliers 355

Contrats de mariage $\quad 211$

Quittances 111

Échanges de terres et de droits successifs immobiliers 65

Obligations $\quad 60$

Inventaires après-décès $\quad 52$

Actes divers concernant les successions 46

Concessions de bancs à l'église 43

Actes divers concernant les affaires seigneuriales

Baux $\quad 34$

Engagements de domestiques 28

Partages $\quad 23$ 
Actes divers concernant les affaires paroissiales

Donations

Remise de terres aux seigneurs $\quad 11$

$\begin{array}{ll}\text { Testaments } & 11\end{array}$

$\begin{array}{ll}\text { Ventes diverses } & 10\end{array}$

Abandons de biens $\quad 7$

Engagements pour les Pays d'en-haut 6

Divers $\quad 56$

Total

De ce tableau se dégagent les principales sphères d'activité de Barette qui nous renseignent sur l'orientation particulière de sa pratique. Le domaine immobilier (concessions, ventes de terres, 91 des 111 quittances, échanges, baux, remises de terres) regroupe 958 actes, soit $60,1 \%$ du total; le droit familial et successoral (contrats de mariage, inventaires après-décès, actes divers touchant les successions, partages, donations, testaments, abandons) concerne 362 actes ou $22,7 \%$ des 1593 contrats que le notaire de La Prairie a préparés. $\mathrm{Au}$ total, ces deux rubriques occupent $82,8 \%$ de toute la pratique de Guillaume Barette.

Ces chiffres appellent quelques commentaires qui expliquent le caractère spécifique de l'étude de Barette. Dans une seigneurie comme celle de La Prairie, deux des principales fonctions de la famille nucléaire, pivot de la société, étaient la constitution et la transmission du patrimoine. Au double problème ainsi posé, les régimes fonciers et successoraux ont apporté des réponses dont témoigne la teneur du greffe de Barette. Dans cet immense territoire seigneurial des jésuites, qui couvrait à peu près $120 \mathrm{~km}^{2}$, le réservoir de terres à concéder était encore énorme au XVIII ${ }^{\text {e }}$ siècle et ne se tarira que dans le premier quart du $\mathrm{XIX}^{\mathrm{e}}$ siècle. Les seigneurs avaient l'obligation de développer leur fief et répondaient, en concédant des terres, à une demande de plus en plus forte au fur et à mesure que la population augmentait au XVIII ${ }^{e}$ siècle. Beaucoup d'habitants étaient déjà propriétaires d'un lopin de terre au moment de leur mariage, et cette assise terrienne constituait l'origine du bien patrimonial que l'on tentait ensuite d'agrandir par d'autres concessions, des achats ou des échanges. À La Prairie, sous le Régime français, à une époque ou l'écoumène était encore vaste, la terre concédée constituait pour la plupart des ménages le principal moyen utilisé pour s'établir. Il n'en sera plus de même au $\mathrm{XIX}^{\mathrm{e}}$ siècle quand presque toutes les terres auront été attribuées et que ce «monde plein» obligera les paysans à déployer des stratégies plus savantes pour assurer leur reproduction sociale. La pratique des 
notaires se conjuguait donc au même temps que cette histoire foncière en mouvement dont a été témoin la vallée du Saint-Laurent entre le $\mathrm{XVII}^{\mathrm{e}}$ et le $\mathrm{XIX}^{\mathrm{e}}$ siècle. La rédaction des actes de concession de terres en particulier meublait une bonne partie de leur pratique sous le Régime français, mais cette activité les occupera de moins en moins au fur et à mesure que l'on avancera dans le XVIII' siècle.

Pour le reste, si l'on excepte les contrats de concession, les ventes et les échanges qui ne sont pas liés à des droits successifs et tous les autres actes relatifs à l'immobilier, le greffe de Barette est surtout composé de contrats concernant le droit familial et successoral. Quand on sait qu'au XVIII ${ }^{e}$ siècle, 95,4\% des habitants de La Prairie demandaient à un notaire de leur préparer un contrat de mariage ${ }^{48}$ pour régler leur communauté de biens et protéger leurs avoirs présents et futurs, il n'est pas étonnant de retrouver dans les minutes de Barette, 211 de ces actes qui représentent $13,2 \%$ du total de sa pratique. Par ailleurs, la transmission des biens, soumise à l'autorité de la coutume de Paris qui instituait l'égalité entre les héritiers et créait de ce fait des situations complexes, a abondamment nourri l'activité notariale. La récurrence de ce souci sans cesse présent dans cette société fondée sur la terre se manifeste à travers les inventaires après-décès, les partages, les accords de succession divers, les ventes et les échanges de parts d'héritage, les donations, les testaments et les abandons. Tous ces actes qui ont trait au domaine successoral rendent compte de la volonté des habitants de se soumettre en général au régime juridique en vigueur dans la colonie en même temps qu'ils témoignent des stratégies utilisées par les donateurs et les rassembleurs pour céder leurs biens fonciers et les remembrer. Il est facile de supposer que le notaire Barette a dû fréquemment assister à de très nombreuses querelles et contestations alimentées par une matière aussi litigieuse.

D'autre part, on peut s'interroger sur la présence discrète dans le greffe de Barette des actes concernant le crédit et le commerce des fourrures. Si les habitants de La Prairie ont peu d'argent à prêter, ils semblent en retour presque tous endettés ainsi que l'attestent les listes de dettes passives qui apparaissent dans les inventaires après-décès. Quand l'endettement est substantiel et le temps du prêt suffisamment long, il est habituellement transcrit dans une obligation rédigée par un notaire. Or, Guillaume Barette ne signe que $20 \%$ à peu près de toutes les obligations qui ont été contractées par les habitants du lieu entre 1709 et 1744 . La soixantaine d'obligations qu'il a préparées impliquaient la plupart du temps de faibles sommes d'argent. En général,

48. Louis Lavallée, La Prairie..., 143. 
elles résultaient d'achats de meubles, de cheptel, de terres, d'arrérages de rentes ou de prêts divers. Les jésuites, souvent présents dans les obligations rédigées par Barette, paraissent les principaux créanciers d'une population qui tarde fréquemment à payer ses redevances à des seigneurs habituellement patients. Dans presque tous les autres cas, faute d'une communauté marchande importante dans la seigneurie des jésuites, c'est vers Montréal que doivent se tourner les habitants de La Prairie pour acheter des marchandises diverses ou emprunter de l'argent. Parce que c'est là que se trouve principalement le capital prêteur détenu par les marchands et les officiers et que résident tous les gros notaires spécialisés dans les opérations de crédit. La ville de Maisonneuve a exercé sur La Prairie, véritable «seigneurie-faubourg», une attraction considérable qui est d'abord de nature économique ${ }^{49}$. Cette soumission économique de La Prairie à Montréal s'exprime donc parfaitement (c'est l'expression la plus manifeste de cette domination) dans les opérations de crédit qui, pour la plupart, sont montréalaises. Cette réalité a limité le champ d'action du notaire Barette et explique la très faible part réservée dans son greffe aux actes de crédit. C'est cette même raison qui rend également compte de l'absence presque totale dans son étude de contrats liés à la traite des fourrures. La Prairie est, au XVIII ${ }^{\mathrm{e}}$ siècle, l'un des premiers réservoirs d'engagés pour la traite aux Pays d'en-haut. Mais cette activité économique appartient d'abord à Montréal, pourvoyeuse de capital qui fait défaut à La Prairie, et à quelques-uns de ses notaires les plus importants spécialisés dans ce genre de pratique ${ }^{50}$. Près de six cents contrats d'engagement pour les Pays d'en-haut dans lesquels apparaissent des habitants de La Prairie ont été rédigés entre 1709 et 1744. De ce nombre, six seulement ont été préparés par Barette et tous les autres par des notaires montréalais. Il y a donc dans cette seule activité notariale, très inégalement partagée entre Montréal et La Prairie, une dimension éloquente des relations ville-campagne et de la domination urbaine. Le contenu du greffe de Guillaume Barette en est la parfaite illustration.

\section{CONCLUSION}

Au terme de cette prospection au cœur de l'activité d'un notaire rural en Nouvelle-France, il est possible de tirer quelques conclusions qui n'ont pas pour but cependant de dresser un type idéal. Guillaume Barette était l'un des notables de la seigneurie de La Prairie et faisait

49. Ibid., 260-262.

50. Gratien Allaire, «Les engagements pour la traite des fourrures: évaluation de la documentation», Revue d'histoire de l'Amérique française, 34,1 (juin 1980): 5-6. 
partie du groupe très restreint de ceux qui savaient lire et écrire. Cette notabilité nous est révélée par le titre qu'il se donnait, le mariage qu'il a contracté avec l'une des familles paysannes les plus en vue de La Prairie, la place réservée qu'il occupait à l'église paroissiale et le rang qu'on lui a reconnu au sein de l'élite qui s'est présentée devant le procureur Collet en 1721. Intermédiaire entre les habitants, les autorités seigneuriales et coloniales, Barette était aussi l'une des figures dominantes de la seigneurie appelée à intervenir fréquemment dans la vie des paysans. Cependant, son statut social au sein de cette société hiérarchisée ne semblait soutenu par aucune puissance économique. Son habitat et sa modeste fortune le situaient aux derniers rangs de l'échelle économique, à la hauteur des paysans les plus médiocres de la seigneurie. Sa profession, dont il vivait sans doute difficilement, l'obligera à devenir paysan ou rentier de la terre et sans doute contrebandier. Pendant toute sa vie, son activité économique sera donc double: à la fois tournée vers la terre et le notariat. Devenu agriculteur, il participe alors au mode de vie le plus commun, celui des paysans, dont il adopte les comportements démographiques et économiques. La dimension de sa famille est à l'image de celle de l'habitant moyen. Et quand il décide (?) de courir les bois et que Pierre et Augustin s'engagent pour monter aux Pays d'en-haut, Guillaume Barette et ses fils ne font que se conformer au modèle qui a tenté bon nombre de paysans de la seigneurie. La conduite qu'adopte Barette dans la constitution de son patrimoine et les manières retenues par le notaire et ses enfants pour le transmettre et le rassembler sont également toutes paysannes.

Son greffe, enfin, est le prisme (un peu déformant toutefois) de la vie des habitants de La Prairie. La pratique de Barette qui éclaire l'histoire interne de la seigneurie s'accordait avec le calendrier agricole, religieux et seigneurial. Plus animée au printemps et à l'été (sauf en août), temps forts de l'activité agricole et seigneuriale, son étude était moins fréquentée l'automne et l'hiver (sauf en novembre) quand la société rurale somnolait et quand le gérant de la seigneurie (un jésuite) devenait moins occupé. Le champ d'action du notaire Barette était cependant réduit, limité pour l'essentiel aux seules affaires qui concernaient la concession des terres, les mutations de propriété, le droit familial et successoral. La majorité des opérations de crédit et presque toutes les transactions liées à la traite aux Pays d'en-haut lui échappaient parce qu'elles étaient réservées aux notaires de Montréal mieux préparés que Barette pour les accueillir. L'étude de la composition du greffe de Guillaume Barette nous aura donc permis finalement de saisir la portée de l'activité d'un notaire rural dont les limites sont révélatrices des relations ville-campagne et de la domi- 
nation que la première exerçait sur la seconde. En ce sens, notre analyse de la pratique de Barette aura été féconde parce qu'elle nous a au moins permis d'éclairer une dimension de l'histoire canadienne mal connue, qui méritait d'être explorée. 